CLINICAL STUDY

\title{
Influence of GH substitution therapy in deficient adults on the recurrence rate of hormonally inactive pituitary adenomas: a case-control study
}

\author{
Michael Buchfelder, Peter Herbert Kann ${ }^{1}$, Christian Wüster ${ }^{2}$, Ulrich Tuschy ${ }^{3}$, Bernhard Saller ${ }^{4}$, Georg Brabant ${ }^{4}$, \\ Andrea Kleindienst and Panagiotis Nomikos and the German KIMS Board \\ Department of Neurosurgery, University of Erlangen-Nuremberg, Schwabachanlage 6, D-91054 Erlangen, Germany, ${ }^{1}$ Division of Endocrinology and \\ Diabetology, University of Marburg, Marburg, D-35032 Germany, ${ }^{2}$ Department of Medicine, University of Heidelberg, Heidelberg, D-61920 Germany, \\ ${ }^{3}$ Department of Medicine, Helios Hospital, D-99089 Erfurt, Germany, ${ }^{4}$ Department of Medicine, University of Essen, D-45122 Essen, Germany and \\ ${ }^{5}$ Department of Medicine, Hannover Medical School, D-30623 Hannover, Germany
}

(Correspondence should be addressed to M Buchfelder; Email: buchfelder@nch.imed.uni-erlangen.de)

\begin{abstract}
Objective: Several studies documented metabolic and psychological benefits of GH substitution in deficient adults, most of them suffering from benign pituitary adenomas. Since GH substitution is considered to promote tumour regrowth, adequate treatment is performed with some reservation. Therefore, we aimed to elucidate the effect of GH replacement therapy on tumour recurrence following surgery.

Methods: In patients with hormonally inactive pituitary adenomas undergoing tumour surgery, a retrospective case-control study was performed. Pre- and postoperative magnetic resonance (MR) images of GH-treated and untreated patients were matched for best fit by two independent observers. The treated patients were retrieved from the surveillance programme of the German KIMS database and the untreated from the database of the Department of Neurosurgery, University of Erlangen. A total of 55 matched pairs were followed for at least 5 years. Tumour recurrence and progression rates were determined according to the postoperative MR.

Results: There were 16 tumour progressions in the treatment group and 12 in the control group. Statistical analysis revealed no significant increase in either recurrence $(P=0.317)$ or progression $(P=0.617)$ within the follow-up period of 5 years when $\mathrm{GH}$ was adequately replaced.

Conclusions: This study provides further observational data of substitution therapy in GH-deficient adults with pituitary adenomas. Comparing long-term surgical results, we found no evidence that GH substitution should be withheld in deficient patients. Even residual tumour does not constitute a contraindication to GH replacement. However, since pituitary tumours are slow growing, an observational period of 5 years may not have been long enough to verify any absolute influence on recurrence potential.
\end{abstract}

European Journal of Endocrinology 157 149-156

\section{Introduction}

Hormonally inactive pituitary adenomas constitute the most frequent cause of growth hormone $(\mathrm{GH})$ deficiency in adulthood. Of all hormonal axes of the hypothalamopituitary system, the somatotroph axis seems to be the most sensitive one to any kind of interference. Thus, most patients with macroadenomas who seek medical care present with insufficient GH secretion and, subsequently, also with inadequate low insulin-like growth factor-I (IGF-I) levels. In several of those patients, in whom the somatotroph axis was still intact, surgery of the tumour may result in GH deficiency (1). Thus, GH substitution therapy is an issue in most patients after surgery of a non-functioning pituitary tumour and has considerable clinical relevance. The clinical impact of GH deficiency in adulthood has been recognized quite recently in the early 1990s, and the benefit of adequate substitution therapy has been appreciated even more lately $(2,3)$. While the advantage of this expensive treatment for metabolism, body composition and psychosocial well-being has been shown convincingly in a number of publications, relevant concern has been raised as to the safety of such a substitution therapy. Large epidemiological observational studies revealed higher mean IGF-I levels in patients who developed prostate, breast and colonic cancer, in comparison with normal controls (4-8). However, all of these IGF-I levels were still within normal limits. On the other hand, acromegalic patients suffer from an increased risk of colonic polyps. Furthermore, several animal studies suggest a 
mitogenic effect of IGF-I. Since surgery of pituitary tumours might leave adenoma tissue residuals, though the remnant may be so small that it escapes the eye of the most expert surgeon, a morphological substrate of a potential threat was anticipated. Thus the objection was that normalized GH and IGF-I levels, such as intended by GH substitution therapy in physiological dosages, could increase the hazard of tumour recurrence and progression respectively.

On this topic, few data are available in the medical literature and the methodology is limited in those already published. Therefore, we performed a case-control study in which we compared matched pairs of 55 patients with hormonally inactive pituitary adenomas, who were either treated or untreated with GH postoperatively. The pairs of patients were selected on the basis of likeness of pre- and postoperative magnetic resonance (MR) images. Among those, we compared tumour recurrence and progression rates over a 5-year period.

\section{Patients and methods}

This study was performed as a non-randomized observational study on two patient groups. The treatment group comprised patients from the German KIMS database, who received GH substitution therapy for GH deficiency in adulthood in different endocrine centres. (KIMS is the Pfizer International Metabolic Database and pharma-epidemilogical survey of adult GH-deficient patients receiving recombinant human GH replacement therapy). The control group comprised patients who had never been exposed to GH therapy and was collected from the database of the Department of Neurosurgery, University of Erlangen-Nuremberg on the basis of the best possible fit matched pairs of pre- and postoperative MR scans of the treated patients.

All patients included in this study previously underwent surgery for a non-functioning pituitary adenoma and the diagnosis was histologically confirmed. There was no evidence of preoperative hormonal hypersecretion in any of the patients. Furthermore, all of them had at least two MR scans, one was obtained not earlier than 2 months after surgery to document the postoperative result and another one at the end of the follow-up period, i.e. 5 years after the initial postoperative MR image. All patients in the control group and 50 out of 55 patients in the treatment group also had a preoperative MR scan that allowed determining the preoperative tumour size. In five patients of the treatment group, the preoperative imaging was not available for analysis. In those, the initial tumour size and extension were estimated from the operation report and from the appearance of the sella in lateral skull X-rays. Generally, T1-weighted images in multiple coronal and sagittal sections were used for analysis. However, magnifications and signal characteristics varied, since the imaging procedures were obtained from different institutions throughout Germany.
Two investigators, blinded to the therapy, analysed the sequential MR images of each pair independently. For every treated patient from the KIMS database, the most similar untreated one was selected from the large database of collected patients from the Department of Neurosurgery, University of Erlangen-Nuremberg, who had an adequate follow-up period and a complete record. Since the tumour size, i.e. the parasellar extension and invasion of the cavernous sinus, determines the surgical resectability and thus constitutes the most important factor for tumour recurrence (9), we matched the patients only on the basis of tumour morphology. In each individual, the volume of suspected tumour tissue was rated as unchanged, larger or smaller in at least two consecutive (or serial) MR studies. In order to allow the most concise and appropriate analysis, the patients were divided into four categories:

Category A (29 patients each group): there was no evidence of residual tumour visible on the postoperative MR scan. The scans were free of artefacts and of ideal quality.

Category B (16 patients each group): there were subtle hints suspicious of residual tumour, such as an asymmetrical cavernous sinus in the postoperative MRI. However, in this group, there was no distinct depiction of residual tumour. In addition, some initial MR scans were difficult to assess because of their suboptimal quality and, thus, were conferred to B.

Category C (eight patients each group): there was unequivocal evidence of small and asymptomatic residual tumour postoperatively.

Category D (two patients each group): there was a large and symptomatic residual tumour. It was expected that an additional treatment was required such as reoperation or irradiation because of the considerable tumour size mass and compression or displacement of adjacent structures.

The treatment group comprised 55 patients who were collected from the German KIMS database, an observational data collection of patients treated with recombinant GH (Genotropin, Pfizer Pharma GmbH, Karlsruhe, Germany). Out of 115 patients having had surgery because of hormonally inactive pituitary adenomas documented in this database, 67 were observed over 60 months after initiation of GH therapy, and they were collected from 20 different neurosurgical centres (Erfurt 4, Mainz 10, Munich 8, Berlin 8, Oldenburg 1, Hamburg 3, Erlangen 2, Heidelberg 6, Hannover 5, Münster 2, Frankfurt 3, Greifswald 3, Leipzig 2, Magdeburg 2, Rostock 1, Düsseldorf 1, Cologne 1, Hagen 1, Freiburg 2, Essen 2). However, only 55 of these patients had MR imaging at least at two time points required for the study, namely after surgery and at the last follow-up visit, 5 years after initiation of $\mathrm{GH}$ substitution therapy.

According to current national criteria, substitution therapy required the documentation of deficient $\mathrm{GH}$ 
secretion by two dynamic tests, during which $\mathrm{GH}$ was supposed to rise to $<3 \mathrm{ng} / \mathrm{ml}$. The time interval between initial surgery and start of GH substitution varied from 0 to 26 years (mean $8.2 \pm 6.7$ years). The initial therapeutic dose of GH varied between 0.4 and 4.0 (mean 1.3) IU per day. The treatment was adjusted to reach age-matched normal IGF-I levels. IGF-I concentrations in 45 out of 55 patients of the GH treatment group were determined in a reference centre in a standardized fashion by a fully automated, two-site chemiluminescent immunoassay (Nichols Advantage; Nichols Institute Diagnostics, San Clemente, CA, USA). Using a multicentre approach, this assay has been found to have a high long-term reproducibility with an estimated coefficient of variation clearly below $7 \%$, while the interindividual biological variation accounts for about 14\% (10). Forty-four of the patients received GH substitution treatment for the entire study period of 5 years. Eleven patients discontinued GH substitution therapy during the observation period for the following reasons: eight of them felt no subjective advantage from treatment, in two patients, other tumours were detected and, in one patient, elevated serum glucose levels were found. Beside the GH deficiency, all but one patient suffered from insufficiencies of other hormonal axes (81\% corticotrophic, $77 \%$ thyrotrophic, $94 \%$ gonadotrophic and $27 \%$ diabetes insipidus). The hormone replacement of other pituitary deficiencies beside $\mathrm{GH}$ was performed in accordance with the individual institutional policy.

The control group was collected from a series of a total of 1179 patients operated on for hormonally inactive pituitary adenomas at the Department of Neurosurgery, University of Erlangen-Nuremberg, Germany. Out of 500 patients with adequate MR scans and a follow-up period of more than 5 years, 55 individuals were selected on the basis of the best possible fit to the GH-treated patients. Since all of the patient pairs were matched on the basis of tumour morphology, the same number of patients was included into the MR categories A-D, both in the treatment and in the control group. However, the demographic data and treatment details turned out to be different in the two groups (Table 1). Forty-five per cent of the control patients suffered from insufficiencies of other hormonal axes ( $24 \%$ corticotrophic, $22 \%$ thyrotrophic, $41 \%$ gonadotrophic and $7.5 \%$ diabetes insipidus).

Statistical analysis was performed by ANOVA and $\chi^{2}$ test with Statgraphics Plus for Windows (StatPoint, Inc., Herndon, VA, USA).

\section{Results}

At the time of surgery, the patients in the treatment group were significantly older than those in the control group $(42.1 \pm 11.2$ respectively $55.5 \pm 13.0$ years, $\left.F_{1,104}=23.19, \quad P<0.001\right)$. At initiation of $\mathrm{GH}$
Table 1 Demographic and clinical data of the two groups of patients: growth hormone-treated patients and untreated controls.

\begin{tabular}{lll}
\hline MR group & $\begin{array}{c}\text { Growth hormone } \\
\text { therapy }\end{array}$ & \multicolumn{1}{c}{ Control group } \\
\hline Invasive parasellar growth & \\
A & $13 / 29(44.8 \%)$ & $13 / 29(44.8 \%)$ \\
B & $8 / 16(50 \%)$ & $8 / 16(50 \%)$ \\
C & $6 / 8(75 \%)$ & $6 / 8(75 \%)$ \\
D & $2 / 2(100 \%)$ & $2 / 2(100 \%)$ \\
Total & $29 / 55(67.5 \%)$ & $29 / 55(67.5 \%)$ \\
Mean age at surgery $($ years \pm s.D.) & \\
A & $42.3 \pm 11.9$ & $53.2 \pm 13.3$ \\
B & $44.1 \pm 9.3$ & $53.4 \pm 13.6$ \\
C & $40.1 \pm 12.5$ & $54.1 \pm 13.8$ \\
D & $29.0 \pm 2.8$ & $56.0 \pm 9.9$ \\
Male:female ratio & $2.3: 1$ & $1.7: 1$ \\
Mean tumour size (mm diameter \pm s.D.) & \\
A & $23.4 \pm 6.8$ & $24.0 \pm 8.7252 .3$ \\
B & $24.8 \pm 4.9$ & $27.8 \pm 7.0112$ \\
C & $28.4 \pm 6.4$ & $29.1 \pm 9.374 .4$ \\
D & $35.0 \pm 4.2$ & $33.5 \pm 6.3$ \\
Total & $28.3 \pm 6.1$ & $28.0 \pm 8.2$ \\
Exclusively primary & $71.0 \%$ & $72.7 \%$ \\
surgery & & \\
Transcranial surgery & $43.6 \%$ & $18.2 \%$ \\
Transsphenoidal & $23.6 \%$ & $27.7 \%$ \\
surgery & & \\
Postoperative & $20.0 \%$ & $23.6 \%$ \\
irradiation & & \\
\hline
\end{tabular}

MR groups: A (no residual tumour), B (subtle hint suspicious for residual tumour), B (asymptomatic residual tumour) and D (symptomatic residual tumour).

replacement, the age of the patients in the treatment group matched the control group's age $(50.6 \pm 9.7$ years, $F_{1,98}=1.57, P=0.214$ ). Any relevant difference in the gender distribution between the two groups was excluded $\left(F_{1,104}=0.38, P=0.538\right)$. A tumour progression was found in a total of 16 patients $(29.1 \%)$ of the treatment group and in 12 patients $(21.8 \%)$ of the control group $(P=0.248)$. In patients without tumour progression, the time interval between surgery and initiation of GH replacement was $9.4 \pm 7.0$ years, while in patients with tumour progression, this time interval was $5.9 \pm 5.6$ years $(P=0.083)$.

The detailed numbers of patients in the different MR categories are given in Figs 1 and 2. In MR category A, 6 out of 29 patients $(20.7 \%)$ in the treatment group suffered recurrences, as opposed to 4 out of 29 patients $(13.7 \%)$ in the control group $(P=0.317)$. The recurrence was defined on the basis of an initial postoperative MR imaging that did not reveal any residual tumour. Three patients in the treatment group and three patients in the control group underwent subsequent irradiation for tumour control. In MR category B, tumour progression was recorded in 5 out of 16 patients $(31.2 \%)$ of the treatment group and in 4 out of 16 patients $(25 \%)$ of the control group $(P=0.617)$. Three patients in the treatment group and four patients in the control group underwent subsequent irradiation. Two examples of MR images of patients with residual 


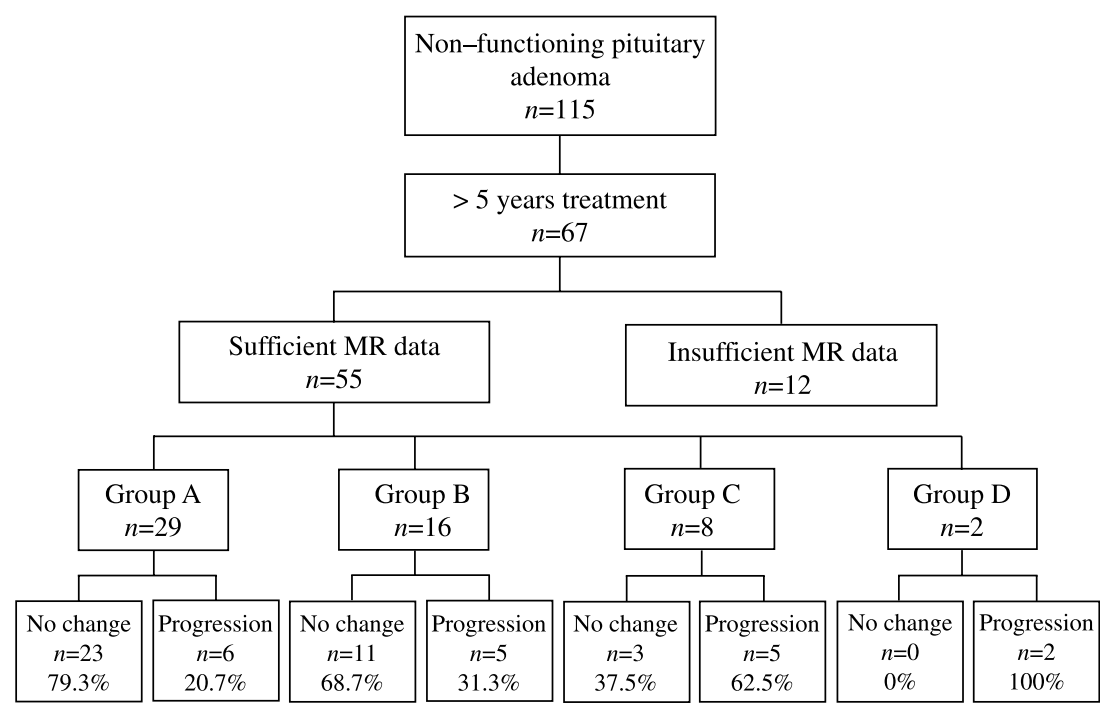

Figure 1 Summary of tumour progression in the four MR-derived categories for the $\mathrm{GH}$-treated patients. MR groups A (no residual tumour), $B$ (subtle hint suspicious for residual tumour), B (asymptomatic residual tumour) and $\mathrm{D}$ (symptomatic residual tumour). tumour, who were treated with GH substitution therapy, are demonstrated in Figs 3 and 4. One patient had a stable tumour for 7 years despite GH treatment (Fig. 3), while the other one developed tumour progression over the 5-year period (Fig. 4). In MR category C, tumour progression was observed in five out of eight patients $(62.5 \%)$ of the treatment group and in four out of eight patients $(50 \%)$ of the control group $(P=0.617)$. Thus, stable tumour was documented in $37.5 \%$ of the treatment group. Three patients in the treatment group and four patients in the control group underwent subsequent irradiation. In MR category D, all tumours progressed, namely two in the treatment and two in the control group. All patients in the treatment and in the control group underwent subsequent irradiation. Despite the fact that in the total series and in all individual MR categories, a few more patients from the treatment group suffered relapses of their tumour growth, neither the total nor any of the differences in the MR subgroups was statistically significant. There was no instance of residual tumour regression observed, neither in the treatment nor in the control group.

In 45 out of $55 \mathrm{GH}$-treated patients in whom the IGFI-levels were monitored routinely in a reference laboratory, mean IGF-I SDS did not differ between the patients with long-term remission and those with tumour progression $(P=0.475)$. In 15 patients of category A, who still had a normal postoperative MR 5 years after initiation of GH treatment, mean IGF-I SDS was $-0.20 \pm 1.34$, and in the patients with progressive tumours, IFG-I SDS was 0.27 \pm 1.01 . Comparably, in category B, mean IGF-I SDS was $0.27 \pm 1.27$ in the patients without evidence of progression and $0.32 \pm$ 1.01 in those whose tumour volumes increased.

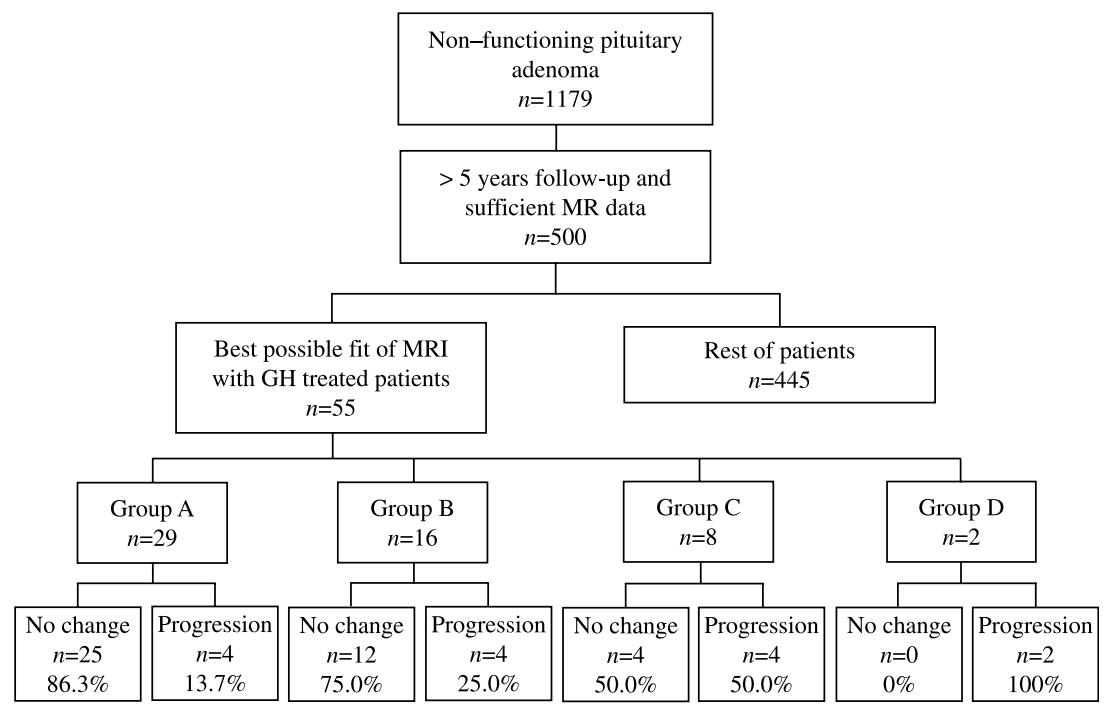

Figure 2 Summary of tumour progression in the four MR-derived categories for the untreated control group. MR groups A (no residual tumour), $B$ (subtle hint suspicious for residual tumour), B (asymptomatic residual tumour) and $\mathrm{D}$ (symptomatic residual tumour). 

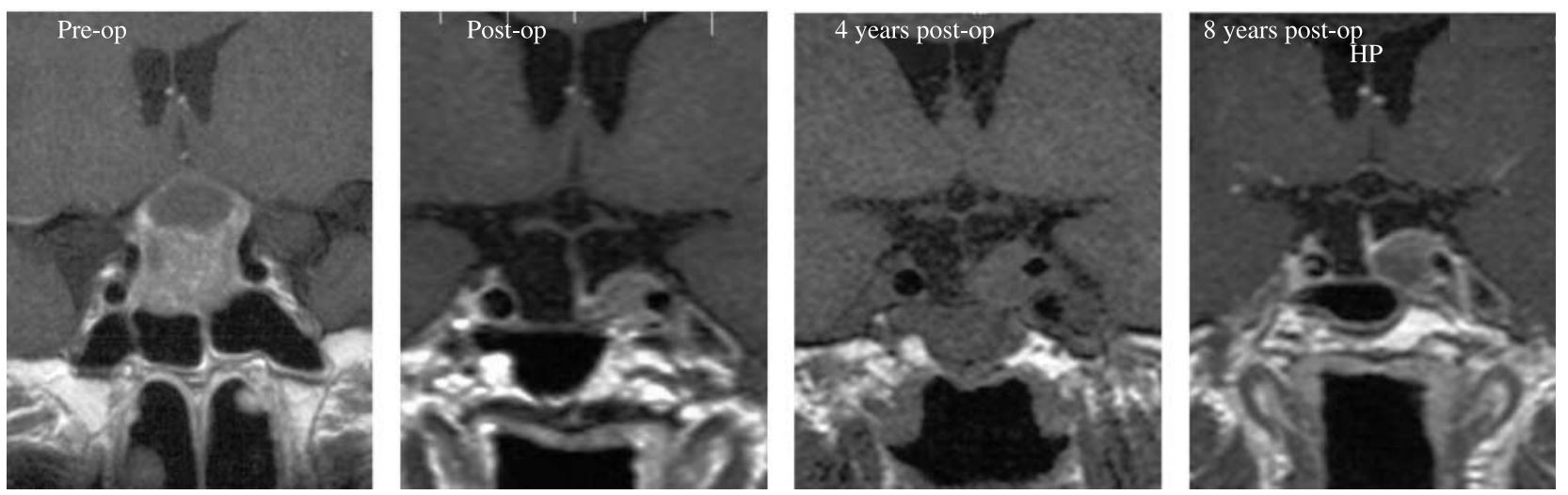

Figure 3 MR depiction of postoperative tumour residual without any regrowth in a patient treated with GH substitution. Coronal MR sections demonstrate the intra- and suprasellar tumour in 1992, which has been operated on through a transsphenoidal approach. However, on the postoperative MR scan in 1993, some remnant tumour is depicted within the cavernous sinus on the right side. In the asymptomatic GH-deficient patient, a GH substitution therapy was started. MR follow-up examinations in 1996 and 2000 reveal no tumour progress over 7 years.

In category $\mathrm{C}$, the patients with stable tumour size had mean IGF-I SDS of $0.13 \pm 1.61$, and in those with tumour progression, mean IGF-I SDS was $1.09 \pm 1.92$. In category $\mathrm{D}$, of the two patients monitored, the mean IGF-I SDS was $-0.94 \pm 0.68$.

\section{Discussion}

The benefit of GH substitution therapy in deficient adults has been demonstrated in a number of studies $(2,3,11)$. However, epidemiological studies that suggest a correlation between the mean IGF-I level and the risk of developing malignant tumours have alerted both the public and the medical experts $(7,8,12)$. Furthermore, individual anecdotal reports related the relapse of an intracranial tumour to $\mathrm{GH}$ treatment $(4,13)$. In contrast to acromegaly, where IGF-I levels are unequivocally elevated and the rate of colonic neoplasms is increased, in GH-deficient patients, the substitution therapy aims at augmentation of hormone levels and normalization of IGF-I. However, the concern on the safety of this therapy remained. Particularly, when residual tumour was present after surgery, one speculated that $\mathrm{GH}$ substitution could promote regrowth of the tumour $(5,6,14)$.

In the literature, essentially three previous studies have assessed a potential influence of GH substitution therapy in deficient adults on the tumour recurrence and progression rate respectively, including miscellaneous types of pituitary adenomas and other neoplasms of the sella turcica region.

Finkenstedt et al. (15) followed six patients for up to 38 months by repeated computed tomography (CT) scanning. Only one of them was irradiated. An increase in tumour size was observed in three of them within 18 months after commencing GH replacement therapy. Tumour volume decreased subsequently in two patients, who were further followed by magnetic resonance imaging. In one patient, tumour enlargement resulted
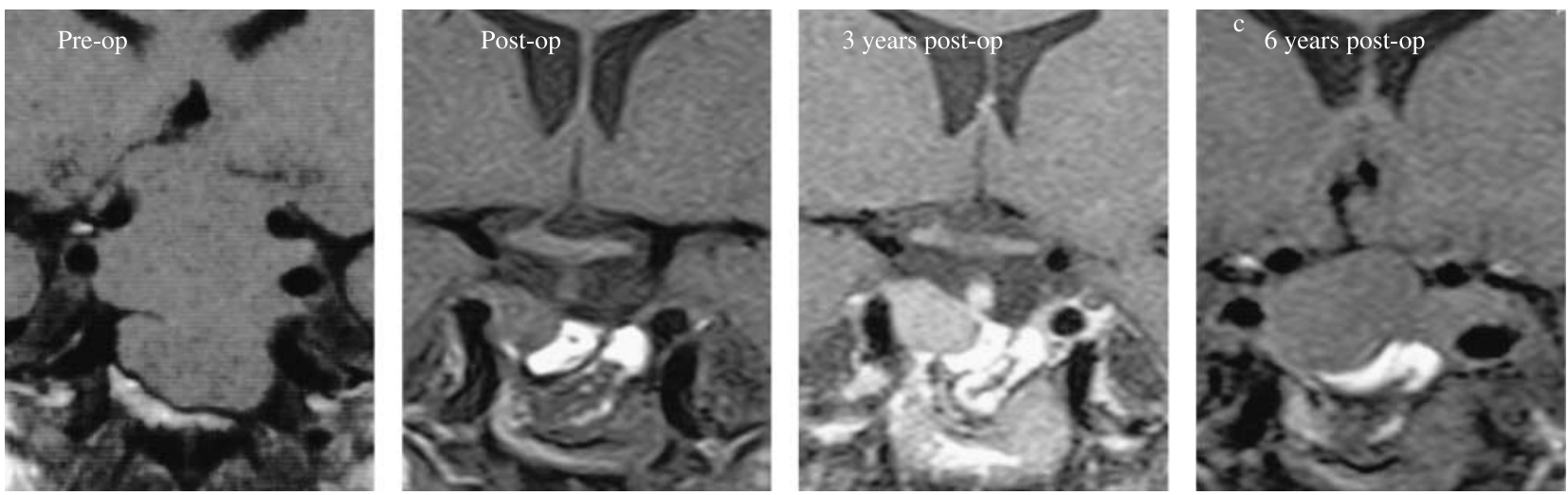

Figure 4 MR depiction of postoperative tumour residual with progression in a patient treated with GH substitution. Coronal MR sections demonstrate the intra- and suprasellar tumour reaching the hypothalamus and compressing the optic chiasm in 1994. The patient has been operated on through a transsphenoidal approach. Postoperatively, the optic chiasm was decompressed, but some residual tumour is depicted in the right cavernous sinus on the postoperative MR in 1995. Owing to GH deficiency, hormone substitution therapy was initiated. MR follow-up examinations in 1997 and 2000 demonstrate tumour recurrence. 
in a discontinuation of the substitution therapy. In all cases with progressive mass lesions, the tumours extended invasively into the cavernous sinus. Five of them were hormonally inactive tumours and one was an folliclestimulating hormone-oma. The authors concluded that their uncontrolled study did not allow determining whether GH therapy promotes tumour growth although their $50 \%$ progression rate appeared to be alarmingly high.

A much larger patient population was studied by Frajese et al. (16). They followed a total of 100 patients and 91 of them after irradiation for at least 12 months. Ninety-two patients were followed-up for 2 years, 63 for 3 years and 23 even for 4 years, most of them by repeated MR scanning. Only six patients had CT studies. In one patient, an increase in intrasellar volume was noted already after 6 months. Since enlargement was considered minor, treatment was not discontinued. Despite ongoing GH therapy, the lesion regressed between the 6th and 12th months of follow-up investigation. The authors reasoned that, at least in irradiated patients, GH substitution does not result in a rapid and substantial increase of tumour size. However, since irradiation has an antiproliferative effect, the effect of $\mathrm{GH}$ substitution on native pituitary adenomas remained still unclear.

Hatrick et al. (14) followed hypopituitary patients with various tumours from an endocrine clinic with respect to potential progression of the neoplasm. However, the authors compared 47 patients treated with recombinant $\mathrm{GH}$ for an average of 3.6 years with 28 controls and found no evidence of increased tumour recurrence in the treatment group. Only 39 patients in the treatment group had GH treatment for more than 2 years. There were different aetiologies and many patients underwent surgery and irradiation.

We felt confident that restricting the study to one pathological aetiology and applying one identical observational period would be superior in elucidating the effective impact of GH treatment. As it is known, in acromegaly and prolactinomas, medical therapy allows not only control of hormone hypersecretion but also reduction of cell proliferation. Thus, in these tumours, the impact of GH may be negligible in the control of tumour regrowth, at least in comparison with the effect of a potent antiproliferative drug treatment with a dopamine agonist. Similarly, serum corticosteroid levels may regulate the proliferation of adrenocorticotrophinsecreting tumours, both in Cushing's disease and in Nelson's syndrome. For these reasons, hormones secreting pituitary adenomas are not the ideal candidates to study the effect of $\mathrm{GH}$ on the proliferation of these tumours. Craniopharyngiomas would certainly be suitable. However, they are even rarer than pituitary adenomas, thus smaller numbers of patients are anticipated resulting in less statistical validity. To pool hormonally inactive adenomas and craniopharyngiomas to answer the problem is also inappropriate since their biological behaviour is completely different.

In both of our groups, the recurrence and progression rates are similar. Due to the fact that we collected the patients from the long-term observational KIMS study and matched the two groups on the basis of preoperative tumour morphology, it resulted in a significant difference in age. Since age at the time of surgery has been found to correlate with cell proliferation and to be a predictor of tumour recurrence in non-functioning pituitary adenomas (9), we cannot exclude an age contribution to our findings. However, the same study found tumour invasion of the cavernous sinus on the preoperative MR scans to be the substantially more important factor for recurrence (9). Furthermore, at the time of initiation of GH replacement, there was no difference in age between the treatment and control groups any longer. Thus, we feel the contribution of age to the recurrence rates found in our study to be negligible.

In the literature, the reported recurrence rate for hormonally inactive tumours varies tremendously, depending on the imaging method used and the duration of the follow-up period (17-20). Depending on the centre, the extent of tumour resection and the follow-up period differed extremely between 8 and $71 \%$. The reported progression rates obviously depend on factors that are related to the tumour, such as size, extension and invasion, and on factors that are related to the subsequent treatment, such as extent of surgery and additional therapies, e.g. conventional external fractionated irradiation or radiosurgery. A retrospective study in macroadenomas reported radiotherapy to improve progression-free survival from 68 to $93 \%$ at 5 years, from 47 to $93 \%$ at 10 years and from 33 to $93 \%$ at 15 years (21). Furthermore, historical series suffer from the disadvantage that without the use of an operating microscope, hardly any tumours could be resected aggressively enough that a follow-up MR investigation would not have revealed evidence of persistent tumour.

Two issues have to be addressed concerning the present study. We compared the surgical long-term results of various specialized and non-specialized surgeons throughout the country with the results in a centre with specific technical expertise. This may be the reason why the results seem to be substantially different, in category A, although the difference does not reach statistical significance. From our surgical experience in Cushing's disease, we have learnt that during surgery, tiny microadenomas are found in about $90 \%$ of cases, despite MR depiction of distinct microadenomas in only about $50 \%$ of cases (22). Thus, the microsurgical sella exploration and aggressive search for abnormal tissues are more sensitive in terms of tumour specimen detection than even the most sophisticated available radiology to date. Although MR imaging is the most 
sensitive tool to decide whether a tumour is completely resected or not, it is not an absolute measure of residual tumour. Postoperative imaging is difficult to interpret, particularly if tissue is implanted to seal the sphenoid sinus $(19,23,24)$. The search for tumour remnants may be more efficient in expert hands, and fewer recurrences would result from more accurate search for tiny tissue residuals. The differing quality of surgical treatment is one inherent bias of the study that seems to influence essentially the results in group A. However, this would not explain a difference in progression of clearly visible tumours, which we did not find actually. In large tumour residuals such as in groups $C$ and $D$, the surgical expertise plays a much lesser role. Large and invasive tumour residuals are the most likely candidates for further progression. Years ago, we could demonstrate that an aggressive tumour growth pattern is associated with an increased proliferative potential of pituitary adenomas (25).

Surprisingly, the recurrence rate found in the present study is unusually high for the standard of modern micro-neurosurgery. Several patients from the KIMS database were operated on many years ago and their treatment may not necessarily reflect modern standards. Together with the fact that the GH-treated patients were collected from a multicentre study including surgically less experienced centres, a higher rate of reoperations was found. Furthermore, one may assume that patients from this inhomogeneous surgical group suffer a higher recurrence rate. Hence, in order to match them with proper controls from the Erlangen database, we had to knowingly choose patients with residual tumour. This negative bias may have accounted for the overall high recurrence rate. In consideration of these factors, our study provides further evidence that GH substitution therapy in GH-deficient adults with an underlying pituitary adenoma is felt a safe treatment. However, in slowly-growing benign tumours, even a follow-up of 5 years may not be adequate, especially since in non-functioning pituitary adenoma, the mean time to tumour growth/recurrence after initial therapy was found to be around 7 years $(17,20)$. Thus, we agree with other investigators that longer follow-up periods are mandatory for a definite statement on the safety of $\mathrm{GH}$ treatment.

\section{Acknowledgements}

This study was supported in part by a grant from Pfizer Pharma GmbH, Karlsruhe, Germany. This manuscript was written on behalf of the members of the German KIMS board: Buchfelder M, Erlangen, Brabant G, Manchester, Fassbender W, Kempen, Faust M, Köln, Forssman K, Karlsruhe, Kann PH, Marburg, Kreitschmann-Andermahr I, Aachen, Koenig J, Karlsruhe, Said E Karlsruche, Tuschy U, Erfurt and Wallaschofski H, Greifswald. The authors thank all the centres (German
KIMS Pharmacogenetics Study Group) contributing data to the German KIMS Pharmacogenetics Study.

\section{References}

1 Losa M, Mortini P, Barzaghi R, Franzin A \& Giovanelli M. Endocrine inactive and gonadotroph adenomas: diagnosis and management. Journal of Neuro-Oncology 200154 167-177.

2 Cuneo RC, Salomon F, Mcgauley GA \& Sonksen PH. The growth hormone deficiency syndrome in adults. Clinical Endocrinology 199237 387-397.

3 Oertel H, Strasburger CJ. Kann P \& Ouabbe HJ. Recommendations for the indications for growth hormone substitution in adult age groups. Working group for pituitary gland and pituitary tumors of the German Society for Endocrinology. Deutsche Medizinische Wochenschrift 1998123 883-886.

4 Ogilvy-Stuart AL, Ryder WD, Gattamaneni HR, Clayton PE \& Shalet SM. Growth hormone and tumour recurrence. BMJ 1992 304 1601-1605.

5 Monson JP. Growth hormone $(\mathrm{GH})$ replacement therapy: cancer in adults treated with GH. Growth Hormone and IGF Research 200010 S50-S51.

6 Chan JM, Stampfer MJ, Giovannucci E, Gann PH, Ma J, Wilkinson P, Hennekens $\mathrm{CH}$ \& Pollak M. Plasma insulin-like growth factor-I and prostate cancer risk: a prospective study. Science 1998279 563-566.

7 Renehan AG, Zwahlen M, Minder C, O'dwyer ST, Shalet SM \& Egger M. Insulin-like growth factor (IGF)-I, IGF binding protein-3, and cancer risk: systematic review and meta-regression analysis. Lancet $20043631346-1353$.

8 Chan JM, Stampfer MJ, Ma J, Gann P, Gaziano JM, Pollak M \& Giovannucci E. Insulin-like growth factor-I (IGF-I) and IGF binding protein-3 as predictors of advanced-stage prostate cancer. Journal of the National Cancer Institute 200294 1099-1106.

9 Losa M, Franzin A, Mangili F, Terreni MR, Barzaghi R, Veglia F, Mortini P \& Giovanelli M. Proliferation index of nonfunctioning pituitary adenomas: correlations with clinical characteristics and long-term follow-up results. Neurosurgery 200047 1318-1319 (discussion 1318-1319).

10 Brabant G, Von Zur Muhlen A, Wuster C, Ranke MB, Kratzsch J, Kiess W, Ketelslegers JM, Wilhelmsen L, Hulthen L, Saller B, Mattsson A, Wilde J, Schemer R \& Kann P. Serum insulin-like growth factor I reference values for an automated chemiluminescence immunoassay system: results from a Multicenter Study. Hormone Research 200360 53-60.

11 Bengtsson BA, Koppeschaar HP, Abs R, Bennmarker H, HernbergStahl E, Westberg B, Wilton P, Monson JP, Feldt-Rasmussen U \& Wuster C. Growth hormone replacement therapy is not associated with any increase in mortality. KIMS Study Group. Journal of Clinical Endocrinology and Metabolism 199984 4291-4292.

12 Hankinson SE, Willett WC, Colditz GA, Hunter DJ, Michaud DS, Deroo B, Rosner B, Speizer FE \& Pollak M. Circulating concentrations of insulin-like growth factor-I and risk of breast cancer. Lancet 1998351 1393-1396.

13 Kiltie AE, Collins CD, Gattamaneni HR \& Shalet SM. Relapse of intracranial germinoma 23 years postirradiation in a patient given growth hormone replacement. Medical and Pediatric Oncology 199729 41-44.

14 Hatrick AG, Boghalo P, Bingham JB, Ayres AB, Sonksen PH \& Russell-Jones DL. Does GH replacement therapy in adult $\mathrm{GH}$-deficient patients result in recurrence or increase in size of pituitary tumours? European Journal of Endocrinology 2002146 807-811.

15 Finkenstedt G, Hofle G, Pallua A, Sailer U \& Gasser RW. Changes in the volume of residual pituitary adenomas in patients with adultonset growth hormone deficiency during replacement therapy with the recombinant human growth hormone. Wiener Klinische Wochenschrift $1999111887-890$. 
16 Frajese G, Drake WM, Loureiro RA, Evanson J, Coyte D, Wood DF, Grossman AB, Besser GM \& Monson JP. Hypothalamo-pituitary surveillance imaging in hypopituitary patients receiving longterm GH replacement therapy. Journal of Clinical Endocrinology and Metabolism 200186 5172-5175.

17 Dekkers OM, Pereira aM, Roelfsema F, Voormolen JH, Neelis KJ, Schroijen MA, Smit JW \& Romijn JA. Observation alone after transsphenoidal surgery for nonfunctioning pituitary macroadenoma. Journal of Clinical Endocrinology and Metabolism 200691 1796-1801.

18 Bradley KM, Adams CB, Potter CP, Wheeler DW, Anslow PJ \& Burke CW. An audit of selected patients with non-functioning pituitary adenoma treated by transsphenoidal surgery without irradiation. Clinical Endocrinology 199441 655-659.

19 Dina TS, Feaster SH, Laws ER, Jr \& Davis DO. MR of the pituitary gland postsurgery: serial MR studies following transsphenoidal resection. American Journal of Neuroradiology 1993 14 763-769.

20 Dekkers OM, Hammer S, De Keizer RJ, Roelfsema F, Schutte PJ, Smit JW, Romijn JA \& Pereira AM. The natural course of nonfunctioning pituitary macroadenomas. European Journal of Endocrinology $2007156217-224$.
21 Gittoes NJ, Bates AS, Tse W, Bullivant B, Sheppard MC, Clayton RN \& Stewart PM. Radiotherapy for non-function pituitary tumours. Clinical Endocrinology 199848 331-337.

22 Buchfelder M, Nistor R, Fahlbusch R \& Huk WJ. The accuracy of CT and MR evaluation of the sella turcica for detection of adrenocorticotropic hormone-secreting adenomas in cushing disease. American Journal of Neuroradiology 199314 1183-1190.

23 Rodriguez O, Mateos B, De La Pedraja R, Villoria R, Hernando JI, Pastor A, Pomposo I \& Aurrecoechea J. Postoperative follow-up of pituitary adenomas after trans-sphenoidal resection: MRI and clinical correlation. Neuroradiology 199638 747-754.

24 Steiner E, Knosp E, Herold CJ, Kramer J, Stiglbauer R, Staniszewski K \& Imhof H. Pituitary adenomas: findings of postoperative MR imaging. Radiology 1992185 521-527.

25 Buchfelder M, Fahlbusch R, Adams EF, Kiesewetter F \& Thierauf P. Proliferation parameters for pituitary adenomas. Acta Neurochirurgica $1996 \mathbf{6 5} 18-21$.

Received 15 March 2007

Accepted 22 May 2007 